\title{
Prevalencia, tratamientos y cuidados de enfermería en la neumonía adquirida en adultos mayores. Revisión sistemática
}

Prevalence, treatments, and nursing care in acquired pneumonia in older adults. Systematic review

Prevalência, tratamento e cuidados de enfermagem em pneumonias adquiridas em adultos idosos. Revisão sistemática

\author{
Rosa Elvira Minchala Urgilés ${ }^{1,2}$ \\ rosaeminchalau@hotmail.com \\ https://orcid.org/0000-0002-0934-9116
}

\author{
Andrés Alexis Ramirez Coronel ${ }^{1,3}$ \\ andres.ramírez@ucacue.edu.ec \\ https://orcid.org/0000-0002-6996-0443
}
Luis Francisco Altamirano Cárdenas ${ }^{1}$
luis.altamirano@ucacue.edu.ec https://orcid.org/0000-0002-7131-9911

\author{
Lilia Azucena Romero Sacoto ${ }^{1}$ \\ lilianrosa72@hotmail.com \\ https://orcid.org/0000-0002-3729-0596
}

\section{María de los Ángeles Estrella González ${ }^{1}$ \\ maria.estrella@ucacue.edu.ec \\ https://orcid.org/0000-0001-7170-7888}

\section{Nancy Isabel Abad Martínez ${ }^{1}$ \\ nancyisabelabadmartínez@yahoo.es \\ https://orcid.org/0000-0002-5888-5521}

\author{
Gloria Luzmila Pogyo Morocho ${ }^{1}$ \\ glorialuzp2010@yahoo.com \\ https://orcid.org/0000-0002-1441-3821 \\ 'Universidad Católica de Cuenca, Sede Azogues. Ecuador \\ ${ }^{2}$ Hospital de Especialidades José Carrasco Arteaga, Cuenca-Ecuador \\ ${ }^{3}$ Laboratorio de Psicometría del Centro de Investigación, Innovación y Transferencia de Tecnología (CIITT) \\ de la Universidad Católica de Cuenca. Ecuador
}

Recibido 6 de septiembre 2021 / Arbitrado y aceptado 27 de octubre 2021 / Publicado 30 de diciembre 2021

\begin{abstract}
RESUMEN
La neumonía es una enfermedad infecciosa adquirida en la comunidad, que afecta principalmente al tracto respiratorio produciendo complicaciones en la vía aérea distal y el parénquima pulmonar. Objetivo. Analizar la prevalencia, tratamientos y cuidados de enfermería, de la neumonía adquirida en la comunidad en los adultos mayores mediante revisión bibliográfica. Metodología. Se trata de un estudio de revisión sistemática, para la búsqueda de información se utilizaron las bases de datos científicas y publicaciones como: Scielo, Elsevier, PubMed, Google académico y otros; publicados en los últimos cinco años, en inglés y español. La búsqueda se realizó mediante la metodología Prisma, en cuatro fases: identificación, selección, elegibilidad, inclusión. Resultados. Se identificaron 1.265 artículos, se seleccionaron 226, de los cuales, se eligieron para la lectura y análisis de 25 artículos y se incluyeron 15 en la muestra final para el estudio; dando como resultado que: la neumonía adquirida en la comunidad (NAC) continúa siendo una de las principales causas de mortalidad, es responsable de 13 de cada 100.000 muertes y del $4 \%$ de egresos hospitalarios. La base para el tratamiento de la NAC son los antibióticos (Amoxicilina, Azitromicina, Claritromicina) y los cuidados de enfermería están centrados en la terapia respiratoria, oxigenoterapia y medidas generales. Conclusiones. La neumonía adquirida en la comunidad afecta principalmente a los adultos mayores de 60 años, produciendo un proceso inflamatorio que compromete a los pulmones. El tratamiento está encaminado con antibióticos, macrólidos, corticoesteroides y es fundamental el cuidado de enfermería, evitando complicaciones y muerte en la población en general.
\end{abstract}

Palabras clave: Neumonía; Participación de la comunidad; Adultos

\begin{abstract}
Pneumonia is a community-acquired infectious disease that mainly affects the respiratory tract, producing complications in the distal airway and lung parenchyma. Objective. Analyze the prevalence, treatment, and nursing care of community-acquired pneumonia in older adults by means of a bibliographic review. Methodology. This is a systematic review study. Scientific databases and publications such as: Scielo, Elsevier, PubMed, academic Google and others were used to search for information; published in the last five years, in English and Spanish. The search was carried out using the Prisma methodology, in four phases: identification, selection, eligibility, inclusion. Results. 1,265 articles were identified, 226 were selected, of which 25 articles were chosen for reading and analysis and 15 were included in the final sample for the study; resulting in: community-acquired pneumonia (CAP) continues to be one of the main causes of mortality, responsible for 13 out of every 100,000 deaths and $4 \%$ of hospital discharges. The basis for the treatment of CAP are antibiotics (Amoxicillin, Azithromycin, Clarithromycin) and nursing care is focused on respiratory therapy, oxygen therapy and general measures. Conclusions. Community-acquired pneumonia mainly affects adults over 60 years of age, producing an inflammatory process that compromises the lungs. Treatment is directed with antibiotics, macrolides, corticosteroids and nursing care is essential, avoiding complications and death in the general population.
\end{abstract}

Key words: Pneumonia, community participation, elderly 


\section{RESUMO}

A pneumonia é uma doença infecciosa adquirida na comunidade que afeta principalmente o trato respiratório, produzindo complicações nas vias aéreas distais e no parênquima pulmonar. Objetivo. Analisar a prevalência, o tratamento e os cuidados de enfermagem da pneumonia adquirida na comunidade em idosos por meio de revisão bibliográfica. Metodologia. Trata-se de um estudo de revisão sistemática, utilizando bases de dados e publicações científicas como: Scielo, Elsevier, PubMed, Google Acadêmico e outros para busca de informações; publicado nos últimos cinco anos, em inglês e espanhol. A busca foi realizada por meio da metodologia Prisma, em quatro fases: identificação, seleção, elegibilidade, inclusão. Resultados. Foram identificados 1.265 artigos, foram selecionados 226, dos quais 25 artigos foram escolhidos para leitura e análise e 15 foram incluídos na amostra final do estudo; resultando em: pneumonia adquirida na comunidade (PAC) continua a ser uma das principais causas de mortalidade, responsável por 13 em cada 100.000 mortes e $4 \%$ das altas hospitalares. A base para o tratamento da PAC são os antibióticos (amoxicilina, azitromicina, claritromicina) e a assistência de enfermagem é voltada para fisioterapia, oxigenoterapia e medidas gerais. Conclusões. A pneumonia adquirida na comunidade afeta principalmente adultos com mais de 60 anos, produzindo um processo inflamatório que compromete os pulmões. $\mathrm{O}$ tratamento é direcionado com antibióticos, macrolídeos, corticosteroides e os cuidados de enfermagem são essenciais, evitando complicações e óbito na população em geral.

Palavras-chave: Pneumonia, participação comunitária, adulto.

\section{INTRODUCCIÓN}

La neumonía adquirida en la comunidad (NAC) es una de las principales enfermedades infecciosas atendidas en los servicios de atención primaria (consultorios y servicios de urgencia), es una causa importante de admisión hospitalaria, ocasionando morbilidad y mortalidad significativa (1). Espín, en su definición presenta como un proceso infeccioso con el cual se complica la vía aérea distal y el parénquima pulmonar, debido al impacto de microorganismos que ingresan a los pulmones, produciendo una respuesta inflamatoria del tracto respiratorio y se puede evidenciar a través de una placa radiológica de infiltrado pulmonar agudo (2). En relación a la etiología, Santana, refiere que "la edad, comorbilidad y factores epidemiológicos, afecta principalmente el parénquima pulmonar y es producida por múltiples microorganismos como bacterias, virus, hongos como: Streptococcus pneumoniae y Mycoplasma pneumoniae y con una menor incidencia son las no infecciosas producidas por aspiración de cuerpos extraños" (3).

Serra et al., manifiestan a la NAC como una enfermedad infecciosa respiratoria que tiene una incidencia promedio a nivel mundial y que oscila entre tres y ocho casos por 1.000 habitantes por año; aumenta con la edad a partir de los 60 años y las comorbilidades propias de este grupo etario. El $40 \%$ de los pacientes ancianos con NAC requieren ingreso hospitalario y alrededor del $10 \%$ necesitan ser admitidos en una Unidad de Cuidados Intensivos ( $\mathrm{UCl}$ ) (4). Espín; en su estudio presenta la mortalidad hospitalaria en el año 2016 por NAC en el Ecuador en los adultos mayores de 80 años con la más alta incidencia llegando a obtener el $54,8 \%$ de los casos de egresos por fallecimiento. La incidencia anual de morbilidad de la NAC en el adulto fluctúa entre 1.07 y 1.2 casos por cada 1.000 personas al año, elevándose en el adulto mayor de 65 años a 12.7-15.3 casos por cada 1.000 personas al año (2). La incidencia es mayor durante la época de invierno.

Se indican las diferentes pautas antibióticas según el ámbito de tratamiento: ambulatorio, hospitalario o Unidad de Cuidados Intensivos y se recomienda calcular la posibilidad de microorganismos resistentes (puntuación PES) (5). En relación a ello, la neumonía se clasifica 
de acuerdo con el tipo de germen que lo causa y el lugar de la infección (6).

Bacterias. La causa más frecuente de neumonía bacteriana es el Streptococcus pneumoniae. Este tipo de neumonía puede producirse sola o después de un resfrío o una gripe, afectando una parte (lóbulo) del pulmón; esta afección se llama neumonía lobar.

Hongos. Es más frecuente en personas con problemas crónicos de salud o un sistema inmunitario debilitado y en personas que han inhalado grandes dosis de estos organismos.

Virus. Algunos de los virus que causan los resfríos y la gripe pueden causar neumonía, siendo estos la causa más frecuente de neumonía en los niños menores de 5 años.

La etiología de la NAC es de capital importancia al momento de tomar las decisiones con respecto a la terapéutica más apropiada (7).

En cuanto a los síntomas asociados a la enfermedad se presentan: Matidez, fiebre, taquipnea, dolor torácico, estertores, egofonía, frémito vocal aumentado y aumento de las vibraciones vocales a la palpación, malestar general, tosconexpectoración(8).Laevaluación de la gravedad permite predecir la evolución de la enfermedad, decidir el lugar de manejo, la extensión del estudio microbiológico y de laboratorio complementario y el tratamiento antimicrobiano empírico (9).

Estudio microbiológico. Facilita a elegir el antibiótico específico, limita la posibilidad de generar resistencia e identificar patógenos de importancia epidemiológica como Streptococcus pneumoniae, Staphylococcus aureus, Legionella pneumophila. El proceso para identificar y aislar los microorganismos se realiza a partir de muestras biológicas como: sangre, expectoración, aspirado traqueal, lavado bronquial por fibrobroncoscopía, líquido pleural, antígeno urinario o pruebas serológicas se reservan para aquellos casos graves o inmunodeprimidos que no responden al tratamiento y se encuentran en las Unidades de Cuidados Intensivos (UCI) (10).

Hemocultivo. Son poco sensibles (4-18\%) pero específicos, discutiendo su relación costeeficacia. El neumococo es el germen aislado en más de la mitad de los casos. Un hemocultivo positivo tiene valor patológico y pronóstico (11).

\section{Complicaciones de la neumonía adquirida en la comunidad}

Las complicaciones se producen cuando la infección no se limita al parénquima pulmonar y se va a otras áreas, cuando la infección es más grande y esto cambia el curso clínico de la neumonía inicial construyendo una hipótesis en su manejo. Las complicaciones son: empiema pulmonar, derrame pleural paraneumónico, absceso pulmonar, neumotórax y fístula broncopleural, las complicaciones son el $1 \%$ de las mismas y se va incrementando a un 40 $\%$ en caso de ingresar al hospital, debido a que se le considera una enfermedad hospitalaria $(12,13)$. 


\section{Tratamiento}

Macrólidos. El uso de macrólidos es una herramienta terapéutica de gran importancia para la NAC, en cuanto garantiza el cubrimiento de gérmenes atípicos. El uso con betalactámicos ha demostrado gran efectividad en la mortalidad 5,25 y se da efectividad o beneficio a los pacientes que están con NAC severa (10).

Corticoesteroides. Su función es inhibir la expresión de citocinas proinflamatorias que están en relación directa con la respuesta inmune dada en la NAC, el uso de corticosteroides no tuvo mucho efecto significativo para la mortalidad, pero sí se encontró una reducción de riesgo en presentar una dificultad respiratoria aguda en los pacientes con NAC (8).

Antibióticos. La exacerbación de la enfermedad pulmonar obstructiva crónica y la neumonía adquirida en la comunidad son las infecciones del tracto respiratorio inferior más frecuentes en la práctica clínica diaria. La selección del antibiótico es un componente crucial en su tratamiento y, en la mayoría de las ocasiones, se realiza de forma empírica. Desde un punto de vista práctico, existen tres claves para la adecuada elección del tratamiento antibiótico oral, que son la efectividad, la seguridad y el impacto ecológico en el microbiota del paciente, incluyendo el desarrollo de resistencias (14).

Se puede mencionar que la utilización de estos tipos de tratamientos farmacológicos dependerá de la edad del paciente, de las comorbilidades asociadas y del lugar de tratamiento, como por ejemplo si es tratado de forma ambulatoria, está en un servicio de hospitalización o en el área de cuidados intensivos. Considerando que la utilización de un tratamiento apropiado reduce la duración de los síntomas, la posibilidad de complicaciones, la estancia hospitalaria y la muerte.

\section{Cuidados de enfermería}

Los cuidados de enfermería van dirigido a todas las edades de los pacientes con neumonía adquirida en la comunidad y sobre todo en los adultos mayores, debido a otras comorbilidades asociadas, a la susceptibilidad a complicaciones y muerte; están encaminadas en relación a: Valorar el estado de hidratación del paciente y cubrir las necesidades si hubiese; Administración del tratamiento según prescripción médica si fuese necesario; Auscultar los pulmones para comprobar la permeabilidad de las vías aéreas; Colocar al paciente en una posición semifowler y ayudarlo a caminar para movilizar las secreciones; Realizar Rx de tórax, gasometría y hemograma para valoración y control; Control y registro de constantes vitales: temperatura, respiración, pulso y tensión arterial; Valorar la saturación de oxígeno, en caso de hipoxemia, suplir la necesidad de oxígeno con cánula nasal en FiO2 menor al 40\%, o mascarilla nasal, si los requerimientos son mayores al 40\%; Valorar el nivel de ansiedad; Terapia respiratoria con ejercicios de estiramiento, tapotaje, tos forzada, inspiración y expiración profunda a través de un inspirómetro; Nebulizaciones con 
broncodilatadores según prescripción médica; Control del estado emocional del paciente y la familia, y Evitar corrientes de aire fría.

La neumonía adquirida en la comunidad es la infección que provoca más ingresos hospitalarios, sobre todo en el caso de la población adulta mayor, ya que son personas extremadamente vulnerables a esta enfermedad y está asociada a otras comorbilidades (diabetes, cardiopatías, neoplasias, desnutrición).

Por lo tanto, es necesario considerar el objetivo del estudio a fin de analizar la prevalencia, los tratamientos farmacológicos y sobre todo enfatizar en los cuidados de enfermería en los adultos mayores, esta profundización de conocimientos se presenta mediante una búsqueda de información científica en las diferentes bases bibliográficas, para contribuir a la capacitación del personal de salud. completa de 15 artículos de forma narrativa, de teoría fundamentada, mediante el estudio documental de revisiones sistemáticas y artículos originales relacionados con el objetivo propuesto. Para la búsqueda de información se utilizaron las bases de datos científicas como: Scielo, Elsevier, PubMed, Google académico y Otros. Como estrategia de búsqueda se utilizaron las palabras clave y conectores: Pneumonia AND Older Adults, Comunity Acquired Pneumonia OR Older Adults, Neumonía AND Síntomas, Pneumonía (OR) Symptoms (AND) Cuidados de enfermería. Se excluyeron: revisiones, artículos o publicaciones duplicadas o de repositorios y que no tengan calidad científica esperada. Para el diseño del análisis de la información se utilizó la metodología Prisma (Figura 1) mediante cuatro frases:

\section{METODOLOGÍA}

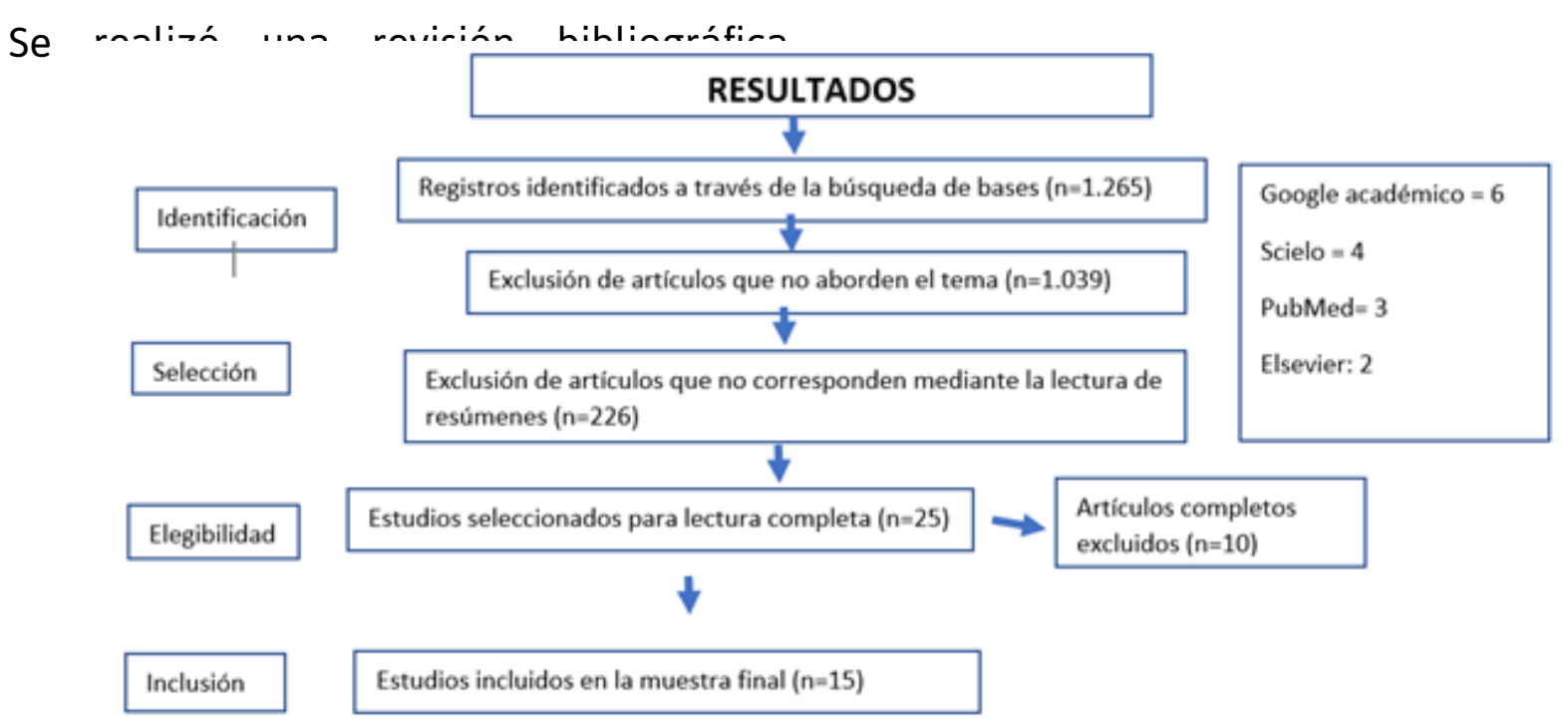

Figura 1. Diagrama de Flujo del proceso de selección de artículos. 
La presentación y análisis se realiza en tablas de distribución de bases científicas encontradas de la metodología utilizada y de acuerdo a las variables planteadas en el objetivo.

\section{DESARROLLO Y DISCUSIÓN}

La neumonía es un proceso inflamatorio agudo del tracto respiratorio y del parénquima pulmonar de origen infeccioso. Los microorganismos pueden llegar al pulmón por vías diferentes: micro aspiraciones de secreciones oro faríngeas (la más frecuente), inhalación de aerosoles contaminados, vía hemática o por contigüidad; y coincide con una alteración de los mecanismos de defensa (mecánicos, humorales o celulares) o con la llegada excesiva de gérmenes que sobrepasan nuestra capacidad normal de "aclaramiento" (11). Es diagnosticada mediante los signos y síntomas, resultados de laboratorio, Radiografía de tórax y en casos necesarios a través de una broncoscopía.

La neumonía adquirida en la comunidad afecta a los dos sexos y a cualquier edad, siendo más susceptibles los adultos mayores y asociados a otras comorbilidades.

Tabla 1. Distribución de Ítems seleccionados por Bases de datos, métodos de estudio y categorías de búsqueda, 2021.

\begin{tabular}{lll}
\hline \multicolumn{1}{c}{ Ítems } & N & $\%$ \\
\hline Base de datos científicas & 5 & 33 \\
Google académico & 4 & 27 \\
Scielo & 4 & 27 \\
PubMed & 2 & 13 \\
Elsevier & & \\
Método de estudio & 7 & 47 \\
$\quad$ Estudios Cuantitativos & 8 & 53 \\
Revisión bibliográfica-sistemática & & \\
Categorías de búsqueda & 5 & 33 \\
$\quad$ Incidencia-prevalencia & 6 & 40 \\
Tratamiento & 4 & 27 \\
Cuidados de enfermería & $\mathbf{1 5}$ & $\mathbf{1 0 0}$ \\
\hline \multicolumn{1}{c}{ Total } & & \\
\hline
\end{tabular}


De lo expuesto en la Tabla 1 , se seleccionaron un total de 15 artículos para la lectura completa y análisis de resultados, publicados en Google académico (33\%), igual porcentaje (27\%) en Scielo y PudMed; del total de artículos revisados, 8 (53\%) corresponden a artículos de análisis bibliográfico y revisión sistemática con metaanálisis y el $47 \%$ son estudios originales con resultados cuantitativos, específicamente estudios probados con diferentes tratamientos para la neumonía adquirida en la comunidad, es por ello, que se presenta el $40 \%$ de artículos que se refieren al tratamiento, el 33\% mencionan sobre la incidencia-prevalencia y el $27 \%$ se enfatiza sobre cuidados de enfermería.

La incidencia anual de morbilidad de la NAC en el adulto mayor fluctúa entre el 1.07 y 1.02 casos por cada 1.000 personas al año (2). La incidencia de neumonía extrahospitalaria (NAC) oscila entre 2-15 casos / 1.000 habitantes / año, siendo mayor en los mayores de 65 años y en pacientes con alta comorbilidad. Alrededor del $75 \%$ de todos los NAC diagnosticados se tratan en el Servicio de Urgencias (SU). Representa la principal causa de sepsis y shock séptico en urgencias, y la causa más frecuente de muerte e ingreso en la Unidad de Cuidados Intensivos ( $\mathrm{UCl}$ ) por enfermedad infecciosa. Entre el 40 y el $60 \%$ de las NAC requerirán ingreso hospitalario, Entre las admisiones, el 2-10\% estará en la UCI (3).

\section{Incidencia y prevalencia}

En el estudio realizado en Chile con el objetivo de elaborar una propuesta para el abordaje de la NAC en el anciano, aplicable tanto en la Atención Primaria de Salud, como en la hospitalización; presentó que la mayoría de los ancianos (259/79 \%) tenían más de 70 años; 266 egresaron vivos y 61 fallecidos. La mortalidad fue de 25,3 x 1000 en el servicio. La letalidad fue del $18,7 \%$; predominó el sexo masculino (4).

Soto en su investigación, con el propósito de determinar la prevalencia de infección por gérmenes atípicos evaluada mediante la determinación de anticuerpos IgM en casos de neumonía adquirida en la comunidad en pacientes adultos inmunocompetentes atendidos en el Hospital Nacional Hipólito Unanue, refiere que se reclutaron 85 pacientes, con la edad promedio de $65,33 \pm 21,43$ años. Se encontraron 3 casos de pacientes con serología positiva a IgM frente a $M$. pneumoniae y 1 caso positivo a $C$. pneumoniae. Los títulos de anticuerpos contra M. pneumoniae tuvieron una correlación altamente significativa con la edad $(r=-0,28 ; p<0,01)$. se encontró una correlación estadísticamente significativa entre los índices de anticuerpos IgM frente a $M$. pneumoniae y $C$. pneumoniae $(r=0,29 ; p<0,01)$ (7). 


\section{Tratamiento}

Los betalactámicos, las fluoroquinolonas y los macrólidos son los fármacos más utilizados por vía oral. El tratamiento de la NAC se fundamenta en la implementación de medidas básicas junto con el manejo antimicrobiano empírico o, en caso de identificar el germen causal, un manejo dirigido. Se recomienda continuar con el uso de macrólidos junto con betalactámicos en neumonía adquirida en la comunidad severa (8).

Están indicados diferentes regímenes de antibióticos, según el ámbito del tratamiento. La duración mínima del tratamiento antibiótico debe ser de 5 días, según criterios de estabilidad clínica. Finalmente, fue revisado la indicación de la vacuna conjugada 13-valente en pacientes inmunocompetentes con factores de riesgo y comorbilidad. Existen vacunas para prevenir algunos tipos de neumonía y la gripe $(5,6)$.

En el caso de pacientes de bajo riesgo de manejo ambulatorio se recomienda tratar con amoxicilina, con o sin inhibidor de B-lactamasas, o macrólidos durante 7-10 días. En los pacientes hospitalizados, se recomienda tratar con agentes -lactámicos asociado a macrólidos o monoterapia con fuoroquinolonas (9). En otro estudio, Salazar, presenta como tratamiento los antibióticos (Amoxicilina, Azitromicina, Claritromicina) pero resulta fundamental considerar la presencia o ausencia de comorbilidades sobre todo en la población adulta mayor (10).

Una pauta empírica inicial, hasta tener los resultados de los cultivos, podría ser la combinación de cefotaxima ( $200 \mathrm{mg} / \mathrm{kg} /$ día) o ceftriaxona $(100 \mathrm{mg} / \mathrm{kg} / \mathrm{día})$ con clindamicina (40 mg/kg/día). Esta última podría retirarse si al recibir el antibiograma se confirma la sensibilidad del germen a la ceftriaxona, e incluso algunas recomendaciones no lo incluyen en la pauta empírica inicial. Como alternativa se podría utilizar amoxicilina-ácido clavulánico (100 mg/kg/día de amoxicilina) (12).

Peñafiel, en el estudio para comparar las características clínicas y de laboratorio en el adulto inmunocompetente hospitalizado por NAC, según el agente causal (S. pneumoniae, microorganismos atípicos y virus respiratorios); con una población de 935 pacientes adultos hospitalizados por NAC, con una media de edad de 69 años, el 51\% de sexo masculino, el 79\% tenía comorbilidades y el $90 \%$ fueron tratados con cefalosporinas de tercera generación asociado a macrólidos (19\%), fluoroquinolonas (21\%) o fármacos antianaerobios (14\%), 43\% fueron manejados en unidad de cuidado intermedio o $\mathrm{UCl}, 11 \%$ requirieron ventilación mecánica, la estadía media en el hospital fueron 9 días; 6,3\% fallecieron en el hospital (1).

La duración del tratamiento dependerá de la evolución clínica, pero generalmente suele ser necesario de 2 a 3 semanas de tratamiento intravenoso, seguido de antibioterapia oral, hasta completar unas 4 semanas. En el 80$90 \%$ responden al tratamiento médico con antibioterapia (12). 


\section{Cuidados de enfermería}

Se consideran los diferentes elementos para una adecuada evaluación y manejo del paciente que cursa con la NAC, el uso de algunos biomarcadores; situaciones especiales para apreciar como la neumonía severa, y varias estrategias para una adecuada prevención.

Las principales medidas de prevención de la neumonía comunitaria incluyen el tratamiento del tabaquismo y los programas de inmunización antiinfluenza y antineumocócica en las poblaciones de riesgo elevado (9). Aligual que, Villasclaras manifiesta que la prevención de las NAC permite actuar directamente contra los patógenos que la causan, mediante la vacunación antineumocócica, efectiva en la prevención de la neumonía neumocócica bacteriémica, y el control medioambiental para la legionella; y de manera indirecta, modificando los factores de riesgo que facilitan las neumonías con el abandono del tabaco que disminuirá el riesgo de sufrir una NAC a la mitad en los 5 años siguientes a dejar el hábito, y la vacunación antigripal, efectiva para prevenir o atenuar la enfermedad viral (11).

La suplencia de oxígeno para lograr una saturación mayor del 90\%, la hidratación y balance electrolítico, la profilaxis para eventos tromboembólicos, terapia respiratoria, manejo de enfermedades concomitantes, monitorización hemodinámica y respiratoria no invasiva para pacientes que se encuentren en la $\mathrm{UCl}$, más reanimación protocolizada (hipoperfusión tisular inducida por sepsis), se constituyen en elementos importantes para el manejo de la patología (8). Así mismo, Villacras recomienda que todos los pacientes con NAC deben tomar líquidos abundantes, hacer reposo en cama mientras tengan fiebre, recibir tratamiento adecuado (antibióticos, antipiréticos), en caso de dolor pleurítico (analgésicos), tos o disnea (nebulizaciones, ambiente húmedo, oxigenoterapia). Los pacientes ingresados pueden requerir oxigenoterapia para mantener una $\mathrm{SpO} 2 \geq$ $90 \%$ o PaO2 $>60 \mathrm{mmHg}$, reposición de líquidos intravenosos, drogas vasoactivas, ventilación mecánica no invasiva o ventilación mecánica invasiva (11).

Se requiere una prevención integral, además de adoptar un estilo de vida saludable para ayudar a mantener fuerte el sistema inmunitario, con lo que es más fácil combatir las enfermedades infecciosas. Para ello, se aconseja seguir una dieta equilibrada en la que no falten nutrientes esenciales, realizar ejercicio moderado de forma habitual, y evitar hábitos nocivos, como el tabaco y el alcohol. Un sistema inmunitario fuerte puede combatir los patógenos que provocan las infecciones en el organismo y evitar que el anciano sufra neumonía (14). A todo ello, se complementa la terapia respiratoria, prevenir tromboembolias de reposo, nebulizaciones, cuidado integral de la piel y evitar infecciones hospitalarias asociadas.

Los ancianos con patologías previas, como EPOC, insuficiencia cardíaca, enfermedad coronaria, diabetes mellitus o enfermedad renal crónica deben acudir de inmediato al servicio de urgencias ante cualquier 
síntoma de neumonía. Es responsabilidad de todos prestar atención a los síntomas de la neumonía, cuidar la alimentación e Higiene de las personas mayores, motivarlas a realizar cualquier tipo de actividad física (moderada), administrar la vacuna antineumocócica y de la gripe y protegerlos de los climas fríos (15). Los antibióticos nuevos junto con la mejora del diagnóstico, relacionados con el uso de ultrasonido pulmonar y pruebas de biomarcadores en el lugar de atención, pueden ayudar a mejorar el tratamiento de la NAC. La prevención, especialmente el uso de la vacuna antineumocócica, es fundamental para reducir la carga de morbilidad y mortalidad (16).

\section{CONCLUSIONES}

La neumonía adquirida en la comunidad afecta principalmente a los adultos mayores de 60 años en adelante, produciendo un proceso inflamatorio, lo cual compromete a las vías respiratorias, al parénquima pulmonar y a los pulmones, como consecuencia de ello, el adulto mayor presenta síntomas como dolor en el pecho, desorientación, fatiga, dificultad para respirar, tos, desaturación de oxígeno y fiebre, de manera que si no son tratados a tiempo puede complicar más su estado se salud y llegar a provocar la muerte.

En relación al tratamiento, existen varios medicamentos con los cuales tratar esta enfermedad entre ellos se encuentran antibióticos, macrólidos, antivirales, antipiréticos, analgésicos y la terapia con corticoesteroides.

Los cuidados que se debe brindar están encaminados a la administración de medicación, de líquidos, el control de las constantes vitales, dieta adecuada, terapia respiratoria y apoyo suplementario de oxígeno.

En relación a lo señalado en el estudio, es necesario, concienciar al paciente y la familia sobre los riesgos de esta enfermedad, a reconocer los signos y síntomas de una posible neumonía para evitar complicaciones o quizá la muerte, y administrar correctamente los medicamentos de acuerdo a la prescripción médica; sobre todo en adultos mayores de 60 años.

\section{REFERENCIAS BIBLIOGRÁFICAS}

1. Peñafiel F, Gassman J, Canelo A, Díaz O. Características clínicas de la neumonía adquirida en la comunidad del adulto inmunocompetente hospitalizado según el agente causal. Rev. médica de Chile; 2018;146(12); 1371-1383. Disponible en: https://scielo.conicyt.cl/scielo.php?script=sci_ arttext\&pid=S0034-98872018001201371

2. Espín A. Neumonía adquirida en la comunidad: valoración inicial y aplicación de escalas de evaluación clínica. Artículos científicos - medicina prehospitalaria. 2019; 3(1): 29-38.

3. Julián-Jiménez A., Adán Valero $Y$, Beteta López A. et.al; Recomendaciones para la atención de pacientes con neumonía extrahospitalaria en el Servicio de Urgencias; Rev. Española de quimioterapia. 2018 Abr; 31 (2): 186-202.

4. Sera $M$, et al. Adulto mayor: propuesta de abordaje de la neumonía adquirida en la comunidad. Artículo especial. [Internet]. 2016 [28 de octubre del 2020]; 14 (2) Disponible en: http://scielo.sld.cu/pdf/ms/v14n2/ms04214. pdf

5. Menéndez R., Catia Cilloniz, Pedro Pablo España, Jordi Almirall, Ane Uranga, Raúl Méndez, David Rigau, Antoni Torres. Neumonía adquirida en la comunidad. Normativa de la Sociedad Española de Neumología y 
Cirugía Torácica (SEPAR). Rev Archivos de bronconeumología 2020, 56(1): 1-10 DOI: 10.1016/j.arbres.2020.01.014

6. MayoClinic. Neumonía. 2017. [Internet]. Estados Unidos: Mayo Foundation for Medical Education and Research: 2018 [citado 28 de octubre del 2020].

7. Soto A., Evaluación de la prevalencia de infección por gérmenes atípicos en pacientes con neumonía adquirida en la comunidad en un hospital de referencia peruano. Rev. De la Facultad de Medicina Humana, 2019 19(4) DOI: 10.25176/RFMH.v19i4.2194

8. Martínez S, Mckinley E, Soto M, Gualtero S. Neumonía adquirida en la comunidad: una revisión narrativa. Universitas Médica. Colombia. 2018 [citado 11 de noviembre del 2020]. 59(4): 93-102. DOI: 10.11144/Javeriana.umed59-4.neum.

9. Fernando Saldías, P., Orlando Díaz P., Evaluación y manejo de la neumonía del adulto adquirida en la comunidad; Rev. Médica Clínica las Condes, 2014, 25(3) 553-564 DOI: 10.1016/S0716-8640(14)70070-7

10. Salazar O. Neumonía adquirida en la comunidad (NAC): Diagnóstico y tratamiento indicado para pacientes adultos mayores. Utmachala [Internet]. 2019 [citado 28 de octubre del 2020]: 60.

11. Villasclaras M, Padilla G, Acosta B. Neumonía adquirida en la comunidad. Re Neumosur 2017: 445-456. Disponible en: https://www. neumosur.net/files/EB03-39\%20NAC.pdf
12. Martín A, Cruz O, Pérez G._Complicaciones de la neumonía adquirida en la comunidad: derrame pleural, neumonía necrotizante, absceso pulmonar y pioneumotórax. Rev. Nemoped. 2017: 1; 127-146. https://www. aeped.es/sites/default/files/documentos/09_ complicaciones_neumonia_adquirida_0.pdf

13. Álvarez $R$, Alos J, Blanquer A, Álvarez J, Garau J, Guerrero Torres A. Guías para el manejo de la neumonía comunitaria del adulto que precisa ingreso en el hospital. Medicina Intensiva.2018;29(1) 24-24.

14. Menéndez R, Cantón R, García A, Barberán J. Tres claves para seleccionar el antibiótico oral adecuado en las infecciones respiratorias; Rev. Española de Quimioterapia; 2019; 32(6): 497-515. Disponible en: https://www.ncbi.nlm. nih.gov/pmc/articles/PMC6913073/

15. Cuidum. Neumonía en ancianos: Complicaciones en la tercera edad. 2021

16. Froes F, Gonçalves Pereira J, Póvoa P; Manejo ambulatorio de la neumonía extrahospitalaria; Rev. Curr Opin Pulm Med; Mayo de 2019; 25 (3): 249-256. DOI: 10.1097 /

MCP. 0000000000000558 .

Conflicto de intereses. Los autores declaran que no existe conflicto de intereses.

Financiación. Este estudio es autofinanciado por los autores.

Agradecimiento. A la Universidad Católica de Cuenca, Sede Azogues y al Laboratorio de Psicometría del Centro de Investigación, Innovación y Transferencia de Tecnología (CIITT) de la Universidad Católica de Cuenca.

\section{ACERCA DE LOS AUTORES}

Rosa Elvira Minchala Urgilés. Licenciada en enfermería. Magíster en Gerencia en Salud para el Desarrollo Local. Magíster en Investigación en Salud, Docente de la Universidad Católica de Cuenca, Sede Azogues-Ecuador.

Lilia Azucena Romero Sacoto. Licenciada en enfermería. Magíster en Gerencia en Salud para el Desarrollo Local. Magister en Gerencia y Liderazgo Educacional. Maestría Gestión del Cuidado. Docente titular auxiliar de la Universidad Católica de Cuenca, Sede AzoguesEcuador.

Andrés Alexis Ramirez Coronel. Psicólogo. Máster en neuropsicología. Doctorado en epidemiologia y bioestadística. Docente de la Maestría en Gestión del Cuidado. Universidad Católica de Cuenca, Sede Azogues-Ecuador.
María de los Ángeles Estrella González. Licenciada en Enfermería. Magíster en Gerencia en Salud para el Desarrollo Local. Docente de la Universidad Católica de Cuenca, Ecuador.

Luis Francisco Altamirano Cárdenas. Médico. Magíster en Crecimiento y Desarrollo. Docente de la Universidad Católica de Cuenca, Ecuador.

Nancy Isabel Abad Martínez. Licenciada en enfermería. Magíster en Gerencia en Salud para el Desarrollo Local. Docente de la Universidad Católica de Cuenca, Sede Azogues-Ecuador

Gloria Luzmila Pogyo Morocho. Licenciada en enfermería. Especialista de Enfermería Clínica. Docente de la Universidad Católica de Cuenca, Sede Azogues-Ecuador. 